\title{
Steady states and nonlinear buckling of cable-suspended beam systems
}

\section{Ivana Bochicchio, Claudio Giorgi \& Elena Vuk}

\section{Meccanica}

An International Journal of Theoretical and Applied Mechanics AIMETA

ISSN 0025-6455

Volume 53

Number 13

Meccanica (2018) 53:3365-3381

DOI 10.1007/s11012-018-0880-9

\section{Meccanica}

An International Journal of Theoretical and Applied Mechanics

\section{AIMETA}

\section{包 Springer}


Your article is protected by copyright and all rights are held exclusively by Springer Nature B.V.. This e-offprint is for personal use only and shall not be self-archived in electronic repositories. If you wish to self-archive your article, please use the accepted manuscript version for posting on your own website. You may further deposit the accepted manuscript version in any repository, provided it is only made publicly available $\mathbf{1 2}$ months after official publication or later and provided acknowledgement is given to the original source of publication and a link is inserted to the published article on Springer's website. The link must be accompanied by the following text: "The final publication is available at link.springer.com". 


\title{
Steady states and nonlinear buckling of cable-suspended beam systems
}

\author{
Ivana Bochicchio · Claudio Giorgi · Elena Vuk
}

Received: 22 November 2017 / Accepted: 11 July 2018/Published online: 19 July 2018

(C) Springer Nature B.V. 2018

\begin{abstract}
This paper deals with the equilibria of an elastically-coupled cable-suspended beam system, where the beam is assumed to be extensible and subject to a compressive axial load. When no vertical load is applied, necessary and sufficient conditions in order to have nontrivial solutions are established, and their explicit closed-form expressions are found. In particular, the stationary solutions are shown to exhibit at most two non-vanishing Fourier modes and the critical values of the axial-load parameter which produce their pitchfork bifurcation (buckling) are established. Depending on two dimensionless parameters, the complete set of resonant modes is devised. As expected, breakdown of the pitchfork bifurcations under perturbation is observed when a distributed transversal load is applied to the beam. In this case, both unimodal and bimodal stationary solutions are studied in detail. Finally, the more complex behavior occurring when trimodal solutions are involved is briefly sketched.
\end{abstract}

\footnotetext{
I. Bochicchio $(\square)$

Dipartimento di Matematica, Università degli studi di

Salerno, Fisciano, Italy

e-mail: ibochicchio@unisa.it

C. Giorgi · E. Vuk

DICATAM, Università degli studi di Brescia, Brescia, Italy
}

Keywords Cable-suspended beam $\cdot$ Suspension bridge $\cdot$ Nonlinear oscillations $\cdot$ Stationary solutions · Pitchfork bifurcation - Biparametric resonance

Mathematics Subject Classification 35B41 . 35Q74 · 74H40

\section{Introduction}

Different kinds of cable-beam models have been proposed and investigated by many researchers not only in the framework of nonlinear dynamics [1-4, 17-20, 29, 30], but also from the mathematical pointview [5, 8, 10, 13, 15, 23, 25-27]. Actually, there are many engineering systems which can be reduced to cable-beam coupled models, cable-stayed bridges and suspension bridges. The beam and the string are two essential structural elements. The analysis of the nonlinear behavior and bifurcations of string-beam coupled systems subjected to parametric excitation are investigated in [4]. The dynamic behavior of models of suspension bridges under different situations was investigated in [1-3], where associated numerical results and physical interpretations were presented. The nonlinear interaction between a beam and a cable in a cable-stayed bridge system was investigated in [17-20], where an analysis of the sensitivity of modal properties to parameter variations has evidenced the occurrence of one-to-two and two-to-one internal 
resonances between global and local modes and the results were verified by both experimental and finite element models.

With the purpose of describing the complex structure of actual bridges, a reliable analytical model should be nonlinear and involve a sufficiently large number of degrees of freedom. This is why the study of such a model requires an extensive use of approximations and numerical simulations (see [1,2]). In order to obtain rigorous results within a precise mathematical setting it is often unavoidable to deal with simpler models. This approach needs some simplifications with respect to the real structure of a single-span suspension bridge. For instance, if the road-bed has sectional dimensions which are negligible in comparison with its length, then it can be simply modeled as a vibrating one-dimensional beam and this entails that the torsional motion can be ignored. In addition, by neglecting the influence of the towers and side parts of the bridge, the beam may be assumed to have simply supported ends. In this connection, the main goal is to construct a class of mathematical models that are quite easy to handle but exhibit some interesting statical and dynamical properties.

\subsection{Motion formulation}

We restrict our attention to a simplified nonlinear cable-suspended beam system. No longitudinal tension is imposed on the cable since we model it as an elastic string with fixed ends. Unlike cable-stayed beam systems, the suspended beam acts on the cable just through the suspenders (a distributed system of linear springs), so yielding a transversal distributed load on it. Of course, this is a simplifying assumption which essentially relies on the straight geometry of the cable at rest. Therefore the road-bed is modeled as an extensible elastic beam which is simply supported at its ends and subject to a constant axial force, whereas the suspension (main) cable behaves as an elastic string with fixed ends (see Fig. 1). A detailed derivation of the model is devised in Sect. 2.1.

Dissipation affecting the cable-suspended beam vibrations may be accounted for as a consequence of different damping mechanisms: internal frictions (within the beam and/or within the cable), damping capacity of the coupling stays, viscous resistance due to the external medium, etc.

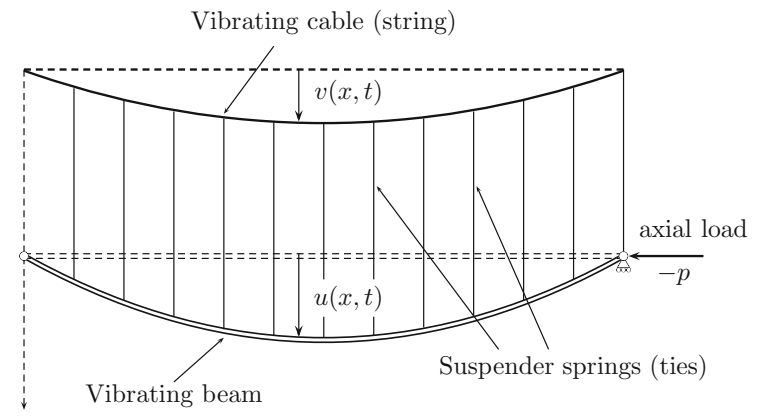

Fig. 1 A simple cable-suspended beam system

Following [3, 9], we model the dynamics of a quite general cable-suspended beam by the following nonlinear system

$$
\left\{\begin{aligned}
\rho_{1} \partial_{t t} u-\varrho \partial_{t t x x} u+\delta_{1} \partial_{x x x x}\left(u+\mu_{1} \partial_{t} u\right)+v_{1} \partial_{t} u \\
-\alpha\left(p+\left\|\partial_{x} u\right\|_{L^{2}(0, \ell)}^{2}\right) \partial_{x x} u+F\left(u-v, \partial_{t}(u-v)\right)=f_{1}, \\
\rho_{2} \partial_{t t} v-\delta_{2} \partial_{x x}\left(v+\mu_{2} \partial_{t} v\right)+v_{2} \partial_{t} v-F\left(u-v, \partial_{t}(u-v)\right)=f_{2},
\end{aligned}\right.
$$

where

- $\quad u=u(x, t):[0, \ell] \times \mathbb{R}^{+} \rightarrow \mathbb{R}$ represents the downward deflection of the beam mid-line in the vertical plane with respect to its reference configuration,

- $\quad v=v(x, t):[0, \ell] \times \mathbb{R}^{+} \rightarrow \mathbb{R}$ measures the vertical displacement of the string,

- $\left\|\partial_{x} u\right\|_{L^{2}(0, \ell)}^{2}$ accounts for the geometric nonlinearity due to the beam elongation,

- $\quad p$ is a real parameter related to the axial force acting at one end of the beam: it is positive when the beam is axially stretched, negative when compressed,

- $F\left(u-v, \partial_{t}(u-v)\right)$ represents the mutual restraining force experienced by both the road bed and the suspension cable as transmitted through the stays: each stay has a rest reference length which corresponds to $u(x)=v(x)=0$,

- $f_{1}$ and $f_{2}$ are the external vertical loads.

All other constants are related to structural parameters of the system, namely

- $\varrho \geq 0$ is the coefficient of rotational inertia,

- $\alpha \geq 0$ is proportional to the axial tension of the beam,

- $\delta_{1}>0$ is the flexural rigidity of the beam, 
- $\delta_{2}>0$ is the coefficient of tensile strength of the cable,

- $\rho_{1}, \rho_{2}>0$ are the mass densities of the beam and the cable, respectively,

- $\mu_{1}, \mu_{2} \geq 0$ represent some internal (Kelvin-Voigt) friction constants,

- $v_{1}, v_{2} \geq 0$ are the coefficients of the linear viscous resistance due to the external medium and acting on the beam and on the cable, respectively.

The unknown fields $u$ and $v$ are required to satisfy the following boundary conditions: the beam is considered with both pinned ends, the string has fixed ends. Accordingly,

$$
\left\{\begin{array}{l}
u(0, t)=u(\ell, t)=\partial_{x x} u(0, t)=\partial_{x x} u(\ell, t)=0, \\
v(0, t)=v(\ell, t)=0
\end{array}\right.
$$

\subsection{Early mathematical contributions}

When a distributed system of one-sided visco-elastic stays is used to connect the beam to the cable, then

$$
F\left(\xi, \partial_{t} \xi\right)=k_{e} \xi^{+}+k_{v} 1_{\xi}^{+} \partial_{t} \xi,
$$

where $k_{e}>0, k_{v} \geq 0$ are the common elastic constant and viscous damping coefficient of the stay system, respectively, and

$$
\xi^{+}=\max \{0, \xi\} \quad, \quad 1_{\xi}^{+}=\xi^{+} / \xi .
$$

This choice leads to a (doubly) nonlinear generalization of the Lazer-McKenna's model

$$
\left\{\begin{aligned}
\rho_{1} \partial_{t t} u-\varrho \partial_{t t x x} u+\delta_{1} \partial_{x x x x}\left(u+\mu_{1} \partial_{t} u\right)+v_{1} \partial_{t} u & \\
\quad-\alpha\left(p+\left\|\partial_{x} u\right\|_{L^{2}(0, \ell)}^{2}\right) \partial_{x x} u+k_{e}(u-v)^{+}+k_{v} 1_{u-v}^{+} \partial_{t}(u-v) & =f_{1}, \\
\rho_{2} \partial_{t t} v- & \delta_{2} \partial_{x x}\left(v+\mu_{2} \partial_{t} v\right)+v_{2} \partial_{t} v-k_{e}(u-v)^{+}-k_{v} 1_{u-v}^{+} \partial_{t}(u-v)=f_{2}
\end{aligned}\right.
$$

Recently, we have dealt with the long-term dynamics of non linear suspended bridges in various works. In [7] we restrict our attention to a simplified non linear uncoupled system where the suspender is replaced by a rigid and unamovable frame (namely, $v=0$ ), the tie lines are only elastic $\left(k_{v}=0\right)$, the rotational inertia $\varrho$ and the internal frictional term $\mu$ are ignored. The asymptotic analysis of the coupled nonlinear system is studied in [8] and [12], with different choices of the parameters $\quad\left(\varrho=\mu_{1}=\mu_{2}=k_{v}=0 \quad\right.$ and $\varrho=v_{1}=v_{2}=k_{v}=0$, respectively). Finally in [9] the longterm behavior of system (1) is analyzed when $\varrho=\mu_{1}=\mu_{2}=0$, whereas $F$ is expressed in a quite general form and the nonlinear term due to extensibility is replaced by a function $M\left(\left\|\partial_{x} u\right\|_{L^{2}(0, \ell)}^{2}\right)$.

When the internal (Kelvin-Voigt) frictions are replaced by linear memory terms of the convolution type and any viscous resistance due to the external medium is neglected, the resulting total dissipation is much weaker. Nevertheless, by neglecting rotational inertia, the existence of a global attractor was proved in [11] for a doubly-nonlinear system where both the extensibility of the beam and the one-sided elastic coupling stays give rise to nonlinear terms. The dynamics of a simpler uncoupled viscoelastic model is analyzed in [10].

On the other hand, when the coupling force $F$ is assumed to be linear in its arguments, the resulting nonlinear dynamics is modeled by the following system

$$
\left\{\begin{array}{l}
\rho_{1} \partial_{t t} u-\varrho \partial_{t t x x} u+\delta_{1} \partial_{x x x x}\left(u+\mu_{1} \partial_{t} u\right)+v_{1} \partial_{t} u \\
\quad-\alpha\left(p+\left\|\partial_{x} u\right\|_{L^{2}(0, \ell)}^{2} \partial_{x x} u+k_{e}(u-v)+k_{v} \partial_{t}(u-v)=f_{1},\right. \\
\rho_{2} \partial_{t t} v-\delta_{2} \partial_{x x}\left(v+\mu_{2} \partial_{t} v\right)+v_{2} \partial_{t} v-k_{e}(u-v)-k_{v} \partial_{t}(u-v)=f_{2} .
\end{array}\right.
$$

When the cable equation is ignored $(v=0)$ and rotational inertia and internal friction vanish $\left(\varrho=v_{1}=0\right)$, the existence of a global attractor is proved in [6]. In the linear elastic homogeneous case $\left(\alpha=\mu_{1}=\mu_{2}=f_{1}=f_{2}=0\right)$, neglecting rotational inertia $(\varrho=0)$, the decay properties of solutions to system (4) have been achieved in [16] by assuming that the viscous force either acts only on the beam $\left(v_{2}=k_{v}=0, v_{1}>0\right)$ or relies only on the damping properties of the coupling stays $\left(v_{1}=v_{2}=0, k_{v}>0\right)$.

\section{A nonlinear cable-suspended beam stationary model}

The global analysis of the longtime dynamics of dissipative systems like (3) and (4), strongly depends on the complexity of the steady-states set. When the dynamical system admits a single stationary solution, then its asymptotical stability is a consequence of the dissipative nature of the problem (either $\mu_{i}>0$ or $\left.v_{i}>0, i=1,2\right)$. In general, when multiple equilibria occur, dissipativity leads the existence of an absorbing set, a closed set which contains all steady states and 
absorbs trajectories in a finite time [22, 24]. In most cases, we are also able to prove the existence of a global regular attractor of solutions. In particular, when a finite set of stationary solutions occurs, the global attractor (if exists) consists of the unstable manifolds connecting them.

This argument motivates the interest to count and possibly construct steady solutions to dissipative systems like (3) and (4). In particular, the number of such solutions strongly depends on the values of the control parameter $p$, in that it is able to induce buckling, a bifurcation in the solution to the equations of static equilibrium [28].

\subsection{Derivation of the dimensionless model}

First, let us consider a thin Woinowsky-Krieger [31] elastic beam of uniform cross section $\Omega$, natural length $\ell$ and thickness $h \ll \ell$. Let assume the beam to be homogeneous, with constant mass density $\rho$ per unit of volume, and symmetric (along with all external loads) with respect to the vertical $x y$-plane, so that we can identify the beam with its rectangular section lying in the plane $z=0$. In the reference configuration, we assume that its middle line occupies the interval $[0, \ell]$ of the $x$-axis. The left end is nailed in its reference configuration at $x=0$, and the right end is displaced in a fixed position $x=\ell+\gamma$, with $\gamma \ll \ell$. From the physical viewpoint, $\gamma<0$ occurs when the beam is axially compressed, $\gamma>0$ when it is subject to traction. Moreover, let $E$ and $v$ be the Young's modulus (force per unit of area) and the Poisson's ratio of the elastic material, respectively. Then, the axial tension acting on $\Omega$ is given by $T=E|\Omega|$, where $|\Omega|$ represents the area of the cross section.

Taking advantage of the analysis carried out in [14] and [21], the (isothermal) motion equation of the beam in the vertical direction turns out to be

$$
\begin{aligned}
& \rho_{h} \partial_{t t}\left(u-\varpi_{h} \partial_{x x} u\right)+\tau \varpi_{h} \partial_{x x x x} u \\
& \quad-\frac{\tau}{\ell}\left[\gamma+\frac{1}{2} \int_{0}^{\ell}\left|\partial_{\xi} u\right|^{2} \mathrm{~d} \xi\right] \partial_{x x} u=\rho_{h} b,
\end{aligned}
$$

where

- $\quad \rho_{h}=m / h(m=\rho|\Omega| \ell)$ is the mass density per unit of thickness,

- $b$ is the vertical body force density per unit of mass,
- $\varpi_{h}=h^{2} / 12$ is the rotational inertia factor,

- $\tau=T /\left(1-v^{2}\right)$.

After eliminating $\rho_{h}$ the motion equation becomes

$$
\begin{aligned}
\partial_{t t} u & -\varpi_{h} \partial_{x x t t} u+\frac{\tau \varpi_{h}}{\rho_{h}} \partial_{x x x x} u \\
& -\frac{\tau}{2 \rho_{h} \ell}\left[2 \gamma+\int_{0}^{\ell}\left|\partial_{\xi} u\right|^{2} \mathrm{~d} \xi\right] \partial_{x x} u=b .
\end{aligned}
$$

The square of the S-wave velocity in bulk elasticity is given by

$$
c_{0}^{2}=\frac{E}{2 \rho(1+v)} \text {. }
$$

Then, we are lead to assume that $\ell$ is the characteristic length and $\ell / c_{0}$ is the characteristic time $t_{0}$ of the problem. Using the dimensionless space and time variables

$$
x^{\star}=\frac{x}{\ell} \in[0,1], \quad t^{\star}=\frac{t}{t_{0}} \in \mathbb{R}^{+},
$$

we introduce the dimensionless unknown

$$
u^{\star}\left(x^{\star}, t^{\star}\right)=\frac{1}{\ell} u\left(\ell x^{\star}, t_{0} t^{\star}\right)
$$

and the following (dimensionless) quantities

$$
\begin{aligned}
& \varpi=\frac{h^{2}}{12 \ell^{2}}, \quad \lambda=\frac{h}{\ell(1-v)}, \\
& \mu=2 \lambda \varpi=\frac{h^{3}}{6(1-v) \ell^{3}}, \\
& \beta=\frac{2 \gamma}{\ell}, \quad b^{\star}\left(x^{\star}, t^{\star}\right)=\frac{2 \rho \ell(1+v)}{E} b\left(\ell x^{\star}, t_{0} t^{\star}\right) .
\end{aligned}
$$

The final form of the motion equation reads (deleting the $\star$ )

$$
\begin{aligned}
\partial_{t t} u & -\varpi \partial_{x x t t} u+\mu \partial_{x x x x} u \\
& -\lambda\left[\beta+\int_{0}^{1}\left|\partial_{\xi} u(\xi, t)\right|^{2} \mathrm{~d} \xi\right] \partial_{x x} u=b .
\end{aligned}
$$

Since $v \in(-1,1 / 2)$ (in particular, $-1<v<0$ for auxetics), all the dimensionless coefficient of (5) are strictly positive, with the exception of the critical parameter $\beta$. Indeed, $\beta<0$ when the beam is axially compressed, whereas $\beta>0$ when it is stretched.

Now, we assume that $b=b_{0}+b_{1}$, where $b_{1}$ is the body force per unit of mass due to the distributed and mutual elastic action exerted between the beam and the suspension cable. It is experienced by a portion of the beam with small thickness, say $h$. Hence, its mass 
is $\rho|\Omega| h$. Then, denoting by $k_{s}$ the common stiffness (force per unit of length) of the suspenders, we obtain $\rho|\Omega| h b_{1}=-k_{s}(u-v)$.

Accordingly, we may represent the dimensionless body force density as follows

$$
\begin{aligned}
b^{\star}\left(x^{\star}, t^{\star}\right) & =b_{0}^{\star}\left(x^{\star}, t^{\star}\right)-\chi\left[u^{\star}\left(x^{\star}, t^{\star}\right)-v^{\star}\left(x^{\star}, t^{\star}\right)\right], \\
\chi & =\frac{2 k_{s} \ell^{2}(1+v)}{E|\Omega| h},
\end{aligned}
$$

so that (5) becomes

$$
\begin{aligned}
& \partial_{t t} u-\varpi \partial_{x x t t} u+\mu \partial_{x x x x} u \\
& \quad-\lambda\left[\beta+\int_{0}^{1}\left|\partial_{\xi} u(\xi, t)\right|^{2} \mathrm{~d} \xi\right] \partial_{x x} u \\
& \quad+\chi(u-v)=b_{0} .
\end{aligned}
$$

Steady states are stationary solutions to (6) and then solve

$$
\begin{aligned}
& \mu \partial_{x x x x} u-\lambda\left[\beta+\int_{0}^{1}\left|\partial_{\xi} u(\xi)\right|^{2} \mathrm{~d} \xi\right] \partial_{x x} u \\
& +\chi(u-v)=b_{0} .
\end{aligned}
$$

After dividing by $\lambda$ we get

$$
\begin{aligned}
& \delta \partial_{x x x x} u-\left[\beta+\int_{0}^{1}\left|\partial_{\xi} u(\xi)\right|^{2} \mathrm{~d} \xi\right] \partial_{x x} u \\
& \quad+k(u-v)=f
\end{aligned}
$$

where

$$
\begin{aligned}
& \delta=\frac{\mu}{\lambda}=2 \varpi=\frac{h^{2}}{6 \ell^{2}}, \\
& k=\chi / \lambda=\frac{2 k_{s} \ell^{3}\left(1-v^{2}\right)}{E|\Omega| h^{2}}, \quad f=b_{0} / \lambda .
\end{aligned}
$$

On the other hand, the cable equation is

$$
\rho_{\ell} \partial_{t t} v-\tau_{\ell} \partial_{x x} v=\rho_{\ell} g,
$$

where $\rho_{\ell}$ and $\tau_{\ell}$ are the mass density and the tension per unit of length, respectively, whereas $g$ is the vertical force per unit of mass. In particular,

$\tau_{\ell}=E_{c}\left|\Omega_{c}\right| / \ell$,

where $E_{c}$ is the Young's modulus and $\Omega_{c}$ the cross section of the cable. By neglecting other contributions, the external vertical force acting on a small cable element with length $h, \rho_{\ell} h g$, must equal the opposite to the elastic action exerted on the beam, namely

$$
\rho_{\ell} h g=k_{s}(u-v),
$$

and then

$$
\partial_{t t} v-\frac{\tau_{\ell}}{\rho_{\ell}} \partial_{x x} v=\frac{k_{s}}{h \rho_{\ell}}(u-v)
$$

In order to rewrite this equation in dimensionless variables, we use the same characteristic length $\ell$ and characteristic time $t_{0}$ as before. Introducing the dimensionless unknown

$$
v^{\star}\left(x^{\star}, t^{\star}\right)=\frac{1}{\ell} v\left(\ell x^{\star}, t_{0} t^{\star}\right),
$$

it follows (deleting the $\star$ )

$$
\begin{aligned}
\partial_{t t} v & -2 \rho(1+v) \frac{\tau_{\ell}}{E \rho_{\ell}} \partial_{x x} v \\
& =2 \rho \ell(1+v) \frac{k_{s}}{E h \rho_{\ell}}(u-v)
\end{aligned}
$$

that in the stationary case reduces to

$$
\eta \partial_{x x} v+k(u-v)=0,
$$

where

$$
\eta=\frac{k h \tau_{\ell}}{k_{s} \ell}=\frac{2 \tau_{\ell} \ell^{2}\left(1-v^{2}\right)}{E|\Omega| h} .
$$

Summarizing, the elastically-coupled cable-beam dimensionless stationary system reads

$$
\left\{\begin{array}{l}
\delta \partial_{x x x x} u-\left[\beta+\int_{0}^{1}\left|\partial_{\xi} u(\xi)\right|^{2} \mathrm{~d} \xi\right] \partial_{x x} u+k(u-v)=f, \\
\eta \partial_{x x} v+k(u-v)=0 .
\end{array}\right.
$$

Coupled systems of this type have been introduced for instance in $[9,12]$.

In order to compare the size of the involved dimensionless parameters, by paralleling (8) we assume

$k_{s}=E_{s}\left|\Omega_{s}\right| / d$,

where $d$ is the free length, $\Omega_{s}$ the cross section and $E_{s}$ the Young's modulus of the suspenders. Accordingly we obtain 


$$
\begin{aligned}
\eta & =\frac{2 E_{c}\left|\Omega_{c}\right| \ell\left(1-v^{2}\right)}{E|\Omega| h}, \\
k & =\frac{2 E_{s}\left|\Omega_{s}\right| \ell^{3}\left(1-v^{2}\right)}{E|\Omega| h^{2} d} .
\end{aligned}
$$

\subsection{The abstract system}

In order to discuss buckling of solutions of system (11), we recall that $\delta, \eta$ and $k$ are positive and dimensionless structural parameters, whereas $\beta \in \mathbb{R}$ is a dimensionless control parameter. The source field $f$ is time-independent, as well as the unknown fields $u$ and $v$ that are required to satisfy the following boundary conditions

$$
\left\{\begin{array}{l}
u(0)=u(1)=\partial_{x x} u(0)=\partial_{x x} u(1)=0, \\
v(0)=v(1)=0 .
\end{array}\right.
$$

Accordingly, the domain of the differential operator $\partial_{x x x x}$ which appears in $(11)_{1}$ is defined as follows,

$$
\begin{aligned}
\mathcal{D}\left(\partial_{x x x x}\right) & =\left\{w \in H^{4}(0,1): w(0)=w(1)\right. \\
& \left.=\partial_{x x} w(0)=\partial_{x x} w(1)=0\right\} .
\end{aligned}
$$

Besides, letting

$$
\mathcal{A}=-\partial_{x x} \quad, \quad \mathcal{D}(\mathcal{A})=H^{2}(0,1) \cap H_{0}^{1}(0,1),
$$

the following peculiar relation holds true

$$
\partial_{x x x x}=\mathcal{A}^{2} \text {. }
$$

$\mathcal{A}$ is a strictly positive selfadjoint operator acting on $L^{2}(0,1)$, with compact inverse. Its discrete spectrum is given by

$$
\lambda_{n}=n^{2} \pi^{2}, \quad n \in \mathbb{N}
$$

and the corresponding eigenvectors are

$$
e_{n}(x)=\sin (n \pi x), \quad n \in \mathbb{N} .
$$

Hence, when conditions (12) are considered, the stationary boundary value problem can be described by means of an abstract system involving a single operator $\mathcal{A}$ which enters the equations at the powers 1 and 2, namely

$$
\left\{\begin{array}{l}
\delta \mathcal{A}^{2} u+\left(\beta+\left\|\mathcal{A}^{1 / 2} u\right\|^{2}\right) \mathcal{A} u+k(u-v)=f, \\
\eta \mathcal{A} v-k(u-v)=0 .
\end{array}\right.
$$

\section{Steady-state solutions}

Let $(H,\langle\cdot, \cdot\rangle,\|\cdot\|)$ be a separable real Hilbert space, and let $\mathcal{A}$ be a strictly positive selfadjoint linear operator on $H$ with domain $\mathcal{D}(A)$ and compact inverse. For $r \in \mathbb{R}$, we define the Hilbert spaces

$$
\begin{array}{cl}
\mathcal{H}^{r}=\mathcal{D}\left(\mathcal{A}^{r / 2}\right), \quad & \langle u, \varphi\rangle_{r}=\left\langle\mathcal{A}^{r / 2} u, \mathcal{A}^{r / 2} \varphi\right\rangle, \\
& \|u\|_{r}=\left\|\mathcal{A}^{r / 2} u\right\| .
\end{array}
$$

Definition 1 Given $f \in \mathcal{H}^{-2}$ and $\beta \in \mathbb{R}$, a pair $(u, v) \in \mathcal{H}^{2} \times \mathcal{H}^{1}$ is a (weak) solution to (13) if

$\left\{\begin{array}{l}\delta\langle u, \varphi\rangle_{2}+\left(\beta+\|u\|_{1}^{2}\right)\langle u, \varphi\rangle_{1}+k\langle u-v, \varphi\rangle=\left\langle\mathcal{A}^{-1} f, \mathcal{A} \varphi\right\rangle, \\ \eta\langle v, \psi\rangle_{1}-k\langle u-v, \psi\rangle=0,\end{array}\right.$

for every $(\varphi, \psi) \in \mathcal{H}^{2} \times \mathcal{H}^{1}$.

Let $\mathcal{S} \subset \mathcal{H}^{2} \times \mathcal{H}^{1}$ be the set of all (weak) solutions to (13). Concerning the existence of solutions, the following result holds.

Theorem 1 (see [9][Th. 2.3]) Given $f \in H^{-2}$ and $\beta \in \mathbb{R}$, the set $\mathcal{S}$ is nonempty and bounded in $\mathcal{H}^{2} \times \mathcal{H}^{1}$.

Remark 1 Due to the structure of the system, if $f \in$ $\mathcal{H}$ and $(u, v)$ is a solution to (13), then $(u, v) \in \mathcal{H}^{4} \times \mathcal{H}^{2}$, and so it is a solution in the strong sense.

The dimensionless, abstract system (13) is relevant in the analysis of the nonlinear buckling problem. The notion of buckling, introduced by Euler more than two centuries ago, describes a static instability of structures due to in-plane loading. In this respect, the main concern is to find the critical buckling loads, that is the threshold value of $\beta$ at which a bifurcation of solutions occurs, and their associated mode shapes, called postbuckling configurations.

\subsection{Free equilibria}

We first scrutinize the homogeneous case, when the transversal load on the beam vanishes, i.e. $f=0$. System (13) reduces to

$\left\{\begin{array}{l}\delta \mathcal{A}^{2} u+C_{u} \mathcal{A} u+k(u-v)=0 \\ \eta \mathcal{A} v-k(u-v)=0\end{array}\right.$ 
where $C_{u}=\beta+\|u\|_{1}^{2}$. According to Remark 1, all solutions are strong. In addition it is easy to prove that every solution can be written as a linear combination of at most two distinct eigenvectors of $\mathcal{A}$.

Lemma 1 Let $(u, v)$ be a solution of system (14). Then

$u=\sum_{n} \alpha_{n} e_{n}, \quad v=\sum_{n} \gamma_{n} e_{n}$,

for some $\alpha_{n}, \gamma_{n} \in \mathbb{R}$, where $\alpha_{n} \neq 0$ for at most two distinct values of $n \in \mathbb{N}$. Moreover,

$\alpha_{n}=0 \quad \Leftrightarrow \quad \gamma_{n}=0$.

Proof By projecting (14) on $e_{n}$, we obtain for every $n \in \mathbb{N}$ the system

$$
\left\{\begin{array}{l}
\delta \lambda_{n}^{2} \alpha_{n}+C_{u} \lambda_{n} \alpha_{n}+k\left(\alpha_{n}-\gamma_{n}\right)=0, \\
\eta \lambda_{n} \gamma_{n}-k\left(\alpha_{n}-\gamma_{n}\right)=0
\end{array}\right.
$$

where $C_{u}=\beta+\sum_{n} \lambda_{n} \alpha_{n}^{2}$. From the second equation it is apparent that

$\gamma_{n}=0 \quad \Leftrightarrow \quad \alpha_{n}=0$.

Substituting the second equation into the first one, we get

$\lambda_{n} \alpha_{n}\left[\delta \lambda_{n}\left(\eta \lambda_{n}+k\right)+C_{u}\left(\eta \lambda_{n}+k\right)+\eta k\right]=0$.

Hence, if $\alpha_{n} \neq 0$ (and so $\gamma_{n} \neq 0$ ), we end up with

$\delta \eta \lambda_{n}^{2}+\left(\eta C_{u}+\delta k\right) \lambda_{n}+k\left(C_{u}+\eta\right)=0$.

Since the equation above admits at most two distinct non-vanishing solutions $\lambda_{n_{i}}$ we are done.

In the sequel we separately scrutinize unimodal (only one eigenfunction is involved) and bimodal (two eigenfunctions are involved) buckled solutions, and we prove that their number depends on structural parameters $(\delta, \eta, k)$ and the applied axial load $\beta$.

\subsubsection{Unimodal solutions}

Let $n \in \mathbb{N}$ be fixed and let

$u=\alpha_{n} e_{n}, \quad v=\gamma_{n} e_{n}$.

After replacing for $u$ and $v$ into system (14), we obtain

$$
\left\{\begin{array}{l}
\delta \lambda_{n}^{2} \alpha_{n}+\left(\beta+\lambda_{n} \alpha_{n}^{2}\right) \lambda_{n} \alpha_{n}+k\left(\alpha_{n}-\gamma_{n}\right)=0, \\
\eta \lambda_{n} \gamma_{n}-k\left(\alpha_{n}-\gamma_{n}\right)=0
\end{array}\right.
$$

where the amplitudes $\alpha_{n}$ and $\gamma_{n}$ are unknown. Substituting the second equation into the first one, we get

$$
\left\{\begin{array}{l}
\lambda_{n} \alpha_{n}\left[\delta \lambda_{n}+\beta+\lambda_{n} \alpha_{n}^{2}+\frac{\eta k}{\left(\eta \lambda_{n}+k\right)}\right]=0, \\
\gamma_{n}=\frac{k \alpha_{n}}{\left(\eta \lambda_{n}+k\right)} .
\end{array}\right.
$$

Looking for nontrivial solutions, we assume $\gamma_{n} \neq 0$ and then we have

$$
\alpha_{n}^{ \pm}= \pm \sqrt{-\delta-\frac{\beta}{\lambda_{n}}-\frac{\eta k}{\lambda_{n}\left(\eta \lambda_{n}+k\right)}}
$$

which are real quantities provided that $\beta<\beta_{n}^{0}$, where

$$
\beta_{n}^{0}(\delta, \eta, k)=-\delta \lambda_{n}-\frac{\eta k}{\eta \lambda_{n}+k}<0, \quad n \in \mathbb{N} .
$$

Hence, buckled solutions of the $n$-th mode occur only if the applied axial compression $-\beta$ exceeds the critical value $-\beta_{n}^{0}$, which depends on the three elastic (dimensionless) parameters of the system: $\delta$ (beam), $\eta$ (cable) and $k$ (suspenders). For later convenience, we introduce the following stiffness ratios,

$\omega=\eta / \delta, \quad \kappa=k / \eta$.

As a consequence, it follows

$\beta_{n}^{0}(\delta, \omega, \kappa)=\delta \sigma_{n}(\omega, \kappa)$,

$\sigma_{n}(\kappa, \omega)=-\lambda_{n}-\frac{\kappa \omega}{\lambda_{n}+\kappa}, \quad n \in \mathbb{N}$.

Unlike $\lambda_{n}$, however, the bifurcation values $\beta_{n}^{0}$ are not ordered by $n$ because of their dependence on $\kappa$ and $\omega$. It is apparent that the occurrence of the same spatial operator, although with different power, in both equations of the system simplifies the problem.

\subsubsection{Resonant set}

Resonant modes arise when two bifurcation values, $\beta_{n}^{0}$ and $\beta_{m}^{0}$ given by (19), overlap for some $n \neq m$. This occurrence depends on two different structural parameters, $\kappa$ and $\omega$.

For any given pair $n, m \in \mathbb{N}, n<m$, let $\mathcal{R}_{n m}$ be the set 


$$
\mathcal{R}_{n m}=\left\{(\kappa, \omega) \in \mathbb{R}^{+} \times \mathbb{R}^{+}: \sigma_{n}(\kappa, \omega)=\sigma_{m}(\kappa, \omega)\right\} .
$$

The resonant condition $\sigma_{n}=\sigma_{m}$, is equivalent to

$$
\begin{gathered}
\kappa^{2}-\left[\omega-\left(\lambda_{n}+\lambda_{m}\right)\right] \kappa+\lambda_{n} \lambda_{m}=0, \\
n, m \in \mathbb{N}, n \neq m .
\end{gathered}
$$

Accordingly, we have $\mathcal{R}_{n m}=\mathcal{R}_{m n}$ and

$$
\begin{aligned}
& \mathcal{R}_{n m}=\left\{(\kappa, \omega) \in \mathbb{R}^{+} \times \mathbb{R}^{+}:\right. \\
& \left.\kappa^{2}-\left[\omega-\left(\lambda_{n}+\lambda_{m}\right)\right] \kappa+\lambda_{n} \lambda_{m}=0\right\} .
\end{aligned}
$$

This set is not empty since the resonant equation has two positive solutions

$$
\begin{aligned}
& \kappa_{n m}^{ \pm}(\omega)=\frac{1}{2}\left\{\omega-\left(\lambda_{n}+\lambda_{m}\right)\right. \\
& \left.\quad \pm \sqrt{\left[\omega-\left(\sqrt{\lambda_{n}}+\sqrt{\lambda_{m}}\right)^{2}\right]\left[\omega-\left(\sqrt{\lambda_{n}}-\sqrt{\lambda_{m}}\right)^{2}\right]}\right\}
\end{aligned}
$$

provided that

$\omega \geq \omega_{n m}=\left(\sqrt{\lambda_{n}}+\sqrt{\lambda_{m}}\right)^{2}$.

It is quite easy to check that three different critical values cannot overlap, whatever $\kappa$ and $\omega$ may be. Then, we define the resonant set $\mathcal{R}$ as follows,

$$
\mathcal{R}=\bigcup_{n<m} \mathcal{R}_{n m}
$$

In Fig. 2 the set $\mathcal{R}$ is depicted by collecting all graphics of $\kappa_{n m}^{ \pm}, n<m$. In particular, the 1:2 resonance is represented by the $\kappa_{12}$ curve, each point of which determines the pair $(\omega, \kappa)$ of structural parameters undergoing a resonant behavior with (eigen)modes 1 and 2.
For any given choice of structural parameters $\omega$ and $\kappa$, condition (20) may be rewritten as

$$
\lambda_{m}=\kappa\left[\frac{\omega}{\lambda_{n}+\kappa}-1\right]
$$

which gives a relation between eigenvalues of the spatial operator yielding a $n: m$ resonance of the system.

\subsubsection{Bimodal solutions}

Let $n, m \in \mathbb{N}, n \neq m$, be fixed and let $u=\alpha_{n} e_{n}+\alpha_{m} e_{m}, \quad v=\gamma_{n} e_{n}+\gamma_{m} e_{m}$

be a solution to (14). Projecting on $e_{n}$ and $e_{m}$, we obtain the following systems

$$
\begin{aligned}
& \left\{\begin{array}{l}
\lambda_{n} \alpha_{n}\left[\delta \lambda_{n}+\beta+\left(\lambda_{n} \alpha_{n}^{2}+\lambda_{m} \alpha_{m}^{2}\right)+\frac{\eta k}{\left(\eta \lambda_{n}+k\right)}\right]=0, \\
\gamma_{n}=\frac{k \alpha_{n}}{\left(\eta \lambda_{n}+k\right)},
\end{array}\right. \\
& \left\{\begin{array}{l}
\lambda_{m} \alpha_{m}\left[\delta \lambda_{m}+\beta+\left(\lambda_{n} \alpha_{n}^{2}+\lambda_{m} \alpha_{m}^{2}\right)+\frac{\eta k}{\left(\eta \lambda_{m}+k\right)}\right]=0, \\
\gamma_{m}=\frac{k \alpha_{m}}{\left(\eta \lambda_{m}+k\right)} .
\end{array}\right.
\end{aligned}
$$

In order to obtain nontrivial amplitudes $\alpha_{n}, \alpha_{m}$ and $\gamma_{n}, \gamma_{m}$ we have to solve the system
Fig. 2 A sketch of the biparametric resonant set $\mathcal{R}$ in the $\omega-\kappa$ plane when $\lambda_{n}=n^{2} \pi^{2}$. The solid curves are the graphics of $\kappa_{n m}^{ \pm}$with $n+m=3,4,5,6,7, n<m$. The limiting curve $\kappa=\omega$ is dashed

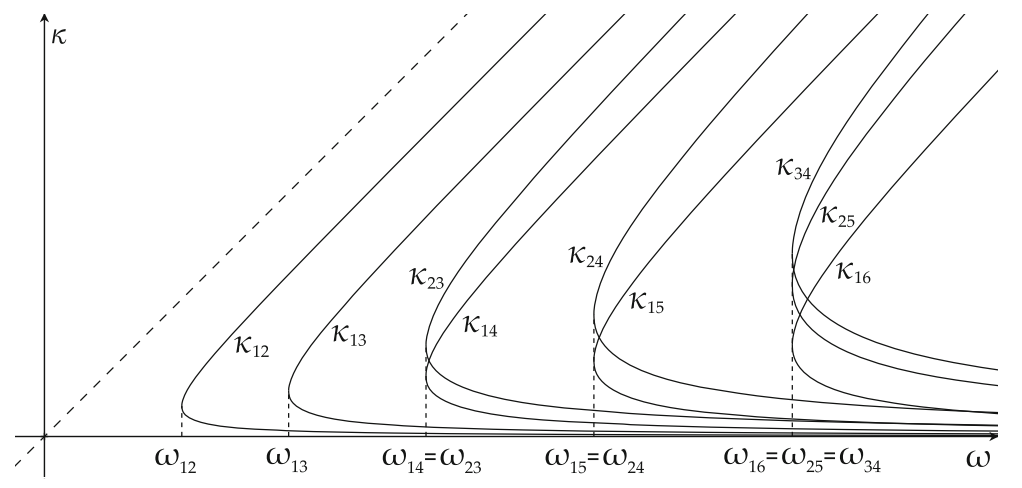




$$
\left\{\begin{array}{l}
\lambda_{n} \alpha_{n}^{2}+\lambda_{m} \alpha_{m}^{2}+\beta=-\delta \lambda_{n}-\frac{\eta k}{\left(\eta \lambda_{n}+k\right)} \\
\lambda_{n} \alpha_{n}^{2}+\lambda_{m} \alpha_{m}^{2}+\beta=-\delta \lambda_{m}-\frac{\eta k}{\left(\eta \lambda_{m}+k\right)} \\
\gamma_{n}=\frac{k \alpha_{n}}{\left(\eta \lambda_{n}+k\right)} \\
\gamma_{m}=\frac{k \alpha_{m}}{\left(\eta \lambda_{m}+k\right)}
\end{array}\right.
$$

From first two equations it is apparent that infinitely many solutions $\alpha_{n}, \alpha_{m}$ do exist provided that their right hand sides are equal. Since $\lambda_{m} \neq \lambda_{n}$, this in turn implies

$$
D_{n m}=\delta\left(\eta \lambda_{n}+k\right)\left(\eta \lambda_{m}+k\right)-\eta^{2} k=0 .
$$

Remark 2 The existence condition $D_{n m}=0$ is equivalent to resonant equation (20) so that inequality (21) may be read as a constraint on the eigenvalues $\lambda_{n}, \lambda_{m}$ which leads to the existence of (infinitely many) bimodal solutions. As a consequence, bimodal solutions are merely resonant solutions, and they occur only if structural parameters satisfy $(\omega, \kappa) \in \mathcal{R}$.

Next subsection is devoted to calculate how many solutions do exist in dependence on the value assumed by the parameter $\beta$.

\subsubsection{Counting of solutions}

Let $n^{\star}$ be the integer valued function given by

$n^{\star}(\beta)=\left|\mathcal{S}^{\star}\right|, \quad \mathcal{S}^{\star}=\left\{n \in \mathbb{N}: \beta>\beta_{n}^{0}\right\}$,

where $|\mathcal{S}|$ stands for the cardinality of the set $\mathcal{S}$. Besides, let

$$
\begin{aligned}
\beta^{\star} & =\max _{n \in \mathbb{N}}\left\{\beta_{n}^{0}\right\}, \\
\beta^{\dagger} & =\max _{n \in \mathbb{N}}\left\{\beta_{n}^{0}: \beta_{n}^{0} \text { is non-simple }\right\} .
\end{aligned}
$$

Of course, $\beta^{\dagger} \leq \beta^{\star}$.

Previous arguments prove the following

Theorem 2 Assume that $(\kappa, \omega) \notin \mathcal{R}$. Then problem (14) has

- only one solution, the straight one $(u, v)=(0,0)$, if $\beta \geq \beta^{\star}$,

- exactly $2 n^{\star}(\beta)+1$ unimodal solutions, the straight one and the buckled pairs,

$$
\begin{aligned}
& u_{n}^{ \pm}(x)=\alpha_{n}^{ \pm} e_{n}, \quad v_{n}^{ \pm}(x)=\frac{\kappa}{\lambda_{n}+\kappa} \alpha_{n}^{ \pm} e_{n}, \\
& \quad n=1,2, \ldots, n^{\star}, \\
& \text { if } \beta<\beta^{\star} .
\end{aligned}
$$

Otherwise, when $(\kappa, \omega) \in \mathcal{R}$ problem (14) has

- only one solution, the straight one $(u, v)=(0,0)$, if $\beta \geq \beta^{\star}$,

- exactly $2 n^{\star}(\beta)+1$ unimodal solutions, the straight one and the buckled pairs, if $\beta^{\dagger}<\beta<\beta^{\star}$,

- infinitely many bimodal solutions if $\beta \leq \beta^{\dagger}$.

3.2 Equilibria under non vanishing distributed transversal load

The next result shows that every solution to the full abstract system (13) can be written as linear combination of at most three distinct eigenvectors of $\mathcal{A}$.

Lemma 2 Let $(u, v)$ be a solution of system (13) with $f=\sum_{n} \phi_{n} e_{n}$. Then

$$
u=\sum_{n} \alpha_{n} e_{n}, \quad v=\sum_{n} \gamma_{n} e_{n},
$$

for some $\alpha_{n}, \gamma_{n} \in \mathbb{R}$, for at most three distinct values of $n \in \mathbb{N}$. Moreover, if $\phi_{n}=0$ then

$\alpha_{n}=0 \quad \Leftrightarrow \quad \gamma_{n}=0$

Proof By projecting (13) on $e_{n}$, we obtain for every $n \in \mathbb{N}$ the system

$$
\left\{\begin{array}{l}
\delta \lambda_{n}^{2} \alpha_{n}+C_{u} \lambda_{n} \alpha_{n}+k\left(\alpha_{n}-\gamma_{n}\right)=\phi_{n}, \\
\eta \lambda_{n} \gamma_{n}-k\left(\alpha_{n}-\gamma_{n}\right)=0 .
\end{array}\right.
$$

From the second equation it is apparent that

$$
\gamma_{n}=0 \quad \Leftrightarrow \quad \alpha_{n}=0
$$

but this occurs only if $\phi_{n}=0$. Substituting the second equation into the first one, we get

$$
\lambda_{n} \alpha_{n}\left[\delta \lambda_{n}\left(\eta \lambda_{n}+k\right)+C_{u}\left(\eta \lambda_{n}+k\right)+\eta k\right]=\left(\eta \lambda_{n}+k\right) \phi_{n}
$$

Hence, we end up with

$$
\begin{aligned}
\delta \eta \alpha_{n} \lambda_{n}^{3} & +\left(\eta C_{u}+\delta k\right) \alpha_{n} \lambda_{n}^{2} \\
& +\left[k\left(C_{u}+\eta\right) \alpha_{n}-\eta \phi_{n}\right] \lambda_{n}-k \phi_{n}=0
\end{aligned}
$$


which admits at most three distinct solutions and then we are done.

In the sequel we separately scrutinize the existence of unimodal, bimodal and trimodal buckled solutions (when one, two or three eigenfunctions are involved, respectively) and their dependence on the applied axial load $\beta$ when structural parameters $\delta, \eta, k$ are fixed.

\subsubsection{Unimodal solutions}

Let $n \in \mathbb{N}$ be fixed and let

$u=\alpha_{n} e_{n}, \quad v=\gamma_{n} e_{n}, \quad f=\phi_{n} e_{n}$,

where $\phi_{n} \neq 0$. After replacing into the abstract system (13) we have

$$
\left\{\begin{array}{l}
\delta \lambda_{n}^{2} \alpha_{n}+\left(\beta+\lambda_{n} \alpha_{n}^{2}\right) \lambda_{n} \alpha_{n}+k\left(\alpha_{n}-\gamma_{n}\right)=\phi_{n}, \\
\eta \lambda_{n} \gamma_{n}-k\left(\alpha_{n}-\gamma_{n}\right)=0 .
\end{array}\right.
$$

Then, substituting the second equation into the first one, we get

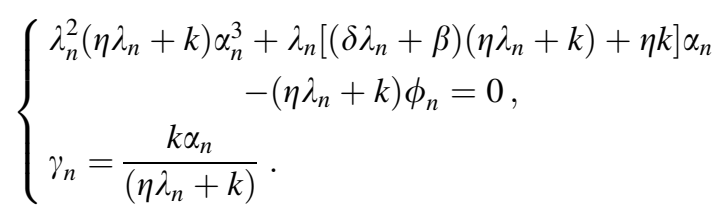

Shortly, the first equation may be rewritten as

$$
\alpha_{n}^{3}+A \alpha_{n}=B
$$

where, accounting for (17),

$$
A=\frac{\beta-\beta_{n}^{0}}{\lambda_{n}}, \quad B=\frac{\phi_{n}}{\lambda_{n}^{2}} .
$$

In order to solve (26), it may be rewritten as

$$
\left\{\begin{array}{l}
y=\alpha_{n}^{3}+A \alpha_{n} \\
y=B
\end{array}\right.
$$

For every values of $B \in \mathbb{R}$ it admits a single solution provided that either $A \geq 0$, or $A<0$ and $2|A| \sqrt{-A}<3 \sqrt{3}|B|$. Summarizing, there exists only one real solution $\alpha_{n}$ (which has the same sign of $\phi_{n}$ ) when

$$
A>-3\left(\frac{B}{2}\right)^{2 / 3} .
$$

On the other hand, if

$$
A \leq-3\left(\frac{B}{2}\right)^{2 / 3}
$$

then (26) admits three real solutions, only one of which has the sign of $\phi_{n}$. Coming back to the original parameters and remembering (17), we conclude that system (25) has

- only one real solution $\left(\alpha_{n}, \gamma_{n}\right)$ if

$$
\beta>\beta_{n}^{\phi}=\beta_{n}^{0}-3\left(\frac{\phi_{n}}{2 \sqrt{\lambda_{n}}}\right)^{2 / 3},
$$

- three real solutions $\left(\alpha_{n}, \gamma_{n}\right)$ if $\beta \leq \beta_{n}^{\phi}$.

It is apparent that $\beta_{n}^{\phi} \leq \beta_{n}^{0}<0$.

When structural parameters $\kappa, \omega, \delta$ are fixed, Eq. (26) provides $\alpha_{n}$ as a function of $\beta$. The resulting perturbed bifurcation of solutions is plotted in Fig. 3, for special values of $\beta_{n}^{0}$ and $\phi_{n}$.

\subsubsection{Bimodal solutions}

Let $n, m \in \mathbb{N}, n \neq m$, be fixed and let $u=\alpha_{n} e_{n}+\alpha_{m} e_{m}, \quad v=\gamma_{n} e_{n}+\gamma_{m} e_{m}, f=\phi_{n} e_{n}+\phi_{m} e_{m}$,

where $\phi_{n}$ and $\phi_{m}$ don't vanish concurrently. We assume that $u, v, f$ satisfy system (13). Then, after projecting each equation of (13) on $e_{n}$ and $e_{m}$ and using (17), we obtain

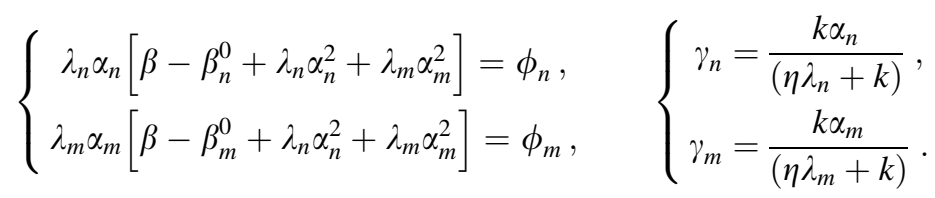


Fig. 3 Perturbed bifurcation of solutions in the non homogeneous case: $\beta_{n}^{0}=-1 / 2, \phi_{n}=-\sqrt{\lambda_{n} / 2}$. The pitchfork curve corresponding to $\phi_{n}=0$ is in gray

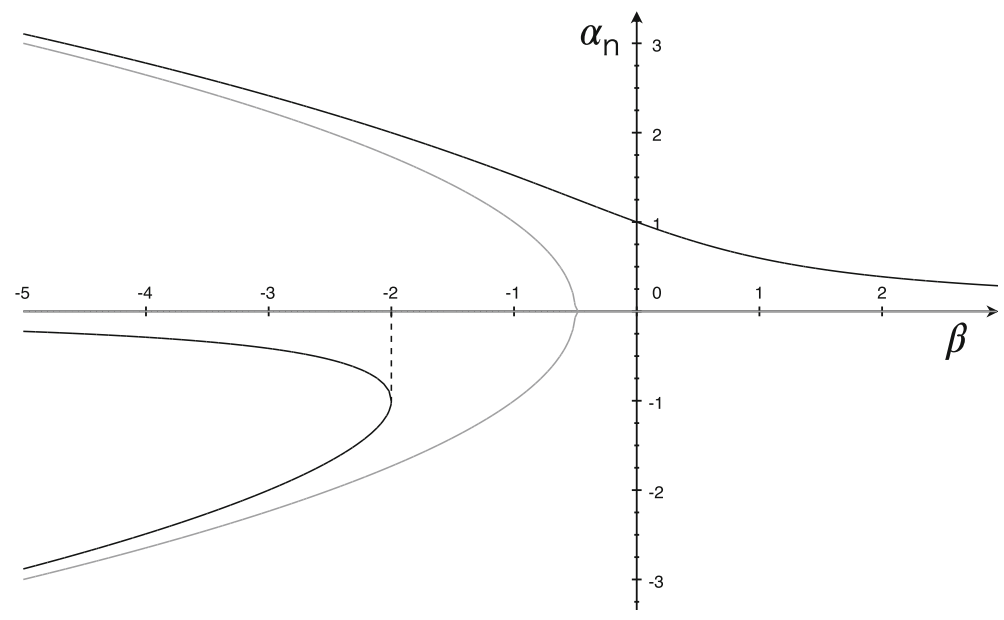

- when $\beta<\tilde{\beta}_{n m}$ there exist three pairs of real solutions $\left(x_{n}, x_{m}\right)$ (Fig. $4 \mathrm{c}$ ).

The approximate value of $\tilde{\beta}_{n m}$ may be obtained numerically or graphically, taking into account that the corresponding cubical curves turn out to be tangent each other, and then tangent to the straight line (29) (Fig. 4b).

As a consequence, the dependence of both $x_{n}$ and $x_{m}$ on the parameter $\beta$ is very close to that depicted in Fig. 3 provided that $\beta_{n}^{\phi}$ is replaced by $\tilde{\beta}_{n m}$.

Otherwise, when $(\kappa, \omega) \notin \mathcal{R}_{n m}$ we have $\beta_{n}^{0} \neq \beta_{m}^{0}$. Then system (28) may be rewritten as follows

$$
\left\{\begin{array}{l}
x_{n}^{3}+x_{n} x_{m}^{2}+\left(\beta-\beta_{n}^{0}\right) x_{n}=\Phi_{n}, \\
\frac{\Phi_{m}}{x_{m}}-\frac{\Phi_{n}}{x_{n}}=\beta_{n}^{0}-\beta_{m}^{0} .
\end{array}\right.
$$

are looking for nontrivial amplitudes $\alpha_{n}, \alpha_{m}$, from $(28)_{1}$ it follows

$$
\Phi_{n} x_{m}=\Phi_{m} x_{n}
$$

When either $\phi_{n}=0$ or $\phi_{m}=0$, then either $x_{n}$ or $x_{m}$ vanishes, so that solutions are no more bimodal. Then, letting $\phi_{n}, \phi_{m} \neq 0$, general solutions $\left(x_{n}, x_{m}\right)$ to system $(28)_{1}$ may be geometrically obtained as intersections of two cubical curves in the $x_{n}-x_{m}$ plane. Because of (29), such intersections, if exist, are aligned. After remembering that

$$
\beta_{j}^{\phi}=\beta_{j}^{0}-3\left(\Phi_{j} / 2\right)^{2 / 3}, \quad j=n, m,
$$

we argue that there exists $\tilde{\beta}_{n m}<\min \left\{\beta_{n}^{\phi}, \beta_{m}^{\phi}\right\}$ such that

- when $\tilde{\beta}_{n m}<\beta \leq \min \left\{\beta_{n}^{\phi}, \beta_{m}^{\phi}\right\}$ only one real pair $\left(x_{n}, x_{m}\right)$ does exist (Fig. 4a);
In the general case, when neither $\phi_{n}$ or $\phi_{m}$ vanish, solutions $\left(x_{n}, x_{m}\right)$ to system (30) may be geometrically obtained as intersections of a cubical curve and an hyperbola in the $x_{n}-x_{m}$ plane. Depending on the choice of structural parameters, there exist at most two critical values, $\hat{\beta}_{n m}$ and $\tilde{\beta}_{n m}$, such that $\hat{\beta}_{n m}<\tilde{\beta}_{n m}<\min \left\{\beta_{n}^{\phi}, \beta_{m}^{\phi}\right\}$ and

- when $\tilde{\beta}_{n m}<\beta \leq \min \left\{\beta_{n}^{\phi}, \beta_{m}^{\phi}\right\}$ only one real pair does exist (Fig. 5a);

- when $\hat{\beta}_{n m}<\beta<\tilde{\beta}_{n m}$ there exist three pairs of real solutions (Fig. 5b);

- when $\beta<\hat{\beta}_{n m}$ there exist five pairs of real solutions (Fig. 5c). 
(a)

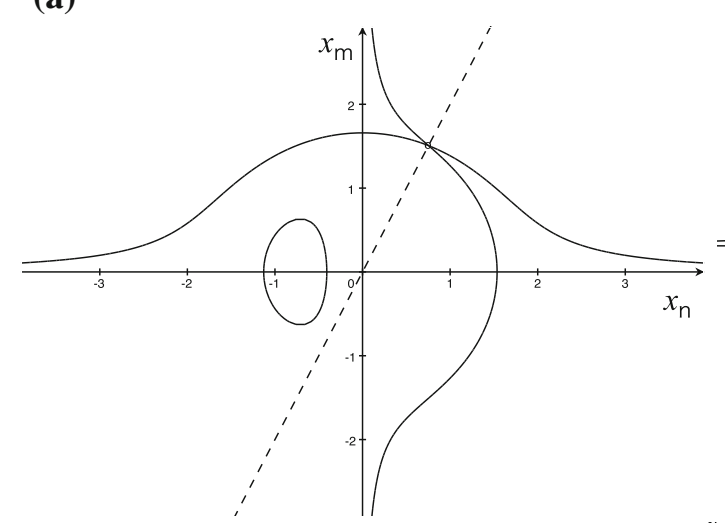

(b)

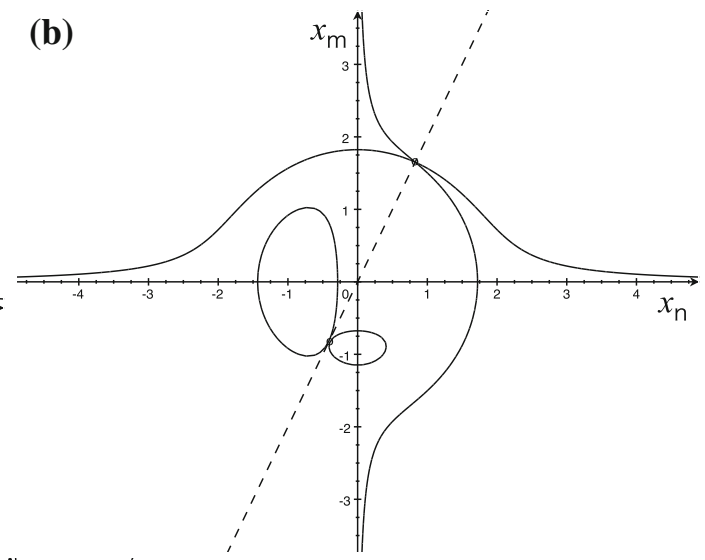

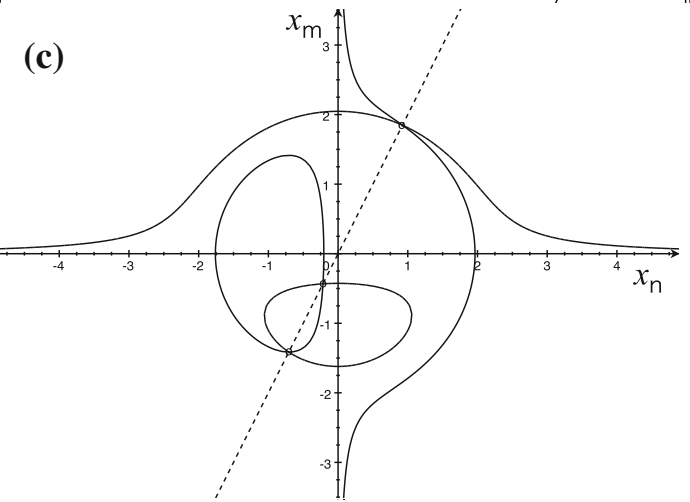

Fig. 4 Solutions $\left(x_{n}, x_{m}\right)$ to system $(28)_{1}$ are represented as intersections (circles) of solid curves. According to (29), they rely on a straight line (dashed). Parameters are such that

For any given choice of the structural parameters and the transversal load, the approximate values of $\tilde{\beta}_{n m}$ and $\hat{\beta}_{n m}$ may be obtained numerically or graphically, taking into account that the corresponding cubical curve turns out to be tangent to either one branch of the hyperbola (Fig. 6).

These results suggest that the dependence of both $x_{n}$ and $x_{m}$ on the parameter $\beta$ has the qualitative behavior depicted in Fig. 7.

In the special case when $\phi_{n}=0$ (30) reduces to

$$
\left\{\begin{array}{l}
{\left[x_{n}^{2}+\beta-\beta_{n}^{0}+\frac{\Phi_{m}^{2}}{\left(\beta_{n}^{0}-\beta_{m}^{0}\right)^{2}}\right] x_{n}=0,} \\
x_{m}=\frac{\Phi_{m}}{\beta_{n}^{0}-\beta_{m}^{0}} .
\end{array}\right.
$$

Thus, $x_{m}$ is independent of $\beta$ whereas $x_{n}$ undergoes a pitchfork bifurcation at $\beta_{n}^{0}=\beta_{m}^{0}=-1 / 2, \quad$ and $\quad \Phi_{m}=2 \Phi_{n}=-\sqrt{2} . \quad$ a $\quad \beta=-2.4$, b $\beta=\tilde{\beta}_{n m} \approx-3.05$ and $\mathbf{c} \beta=-4$

$\beta=\beta_{n}^{0}-\frac{\Phi_{m}^{2}}{\left(\beta_{n}^{0}-\beta_{m}^{0}\right)^{2}}<\beta_{n}^{0}$.

When $\phi_{m}=0$ the same conclusion occurs, but indexes $n$ and $m$ exchange.

\subsubsection{Trimodal solutions}

Let $n, m, s \in \mathbb{N}, n \neq m \neq s$, be fixed and let $f=\phi_{n} e_{n}+\phi_{m} e_{m}+\phi_{s} e_{s}$

be the the distributed transversal load, where $\phi_{n}, \phi_{m}$ and $\phi_{s}$ don't vanish concurrently. According to Lemma 2 we are looking for general solutions to (13) in the form

$u=\alpha_{n} e_{n}+\alpha_{m} e_{m}+\alpha_{s} e_{s}, \quad v=\gamma_{n} e_{n}+\gamma_{m} e_{m}+\gamma_{s} e_{s}$.

After projecting each equation of (13) on $e_{n}, e_{m}$ and $e_{s}$, 

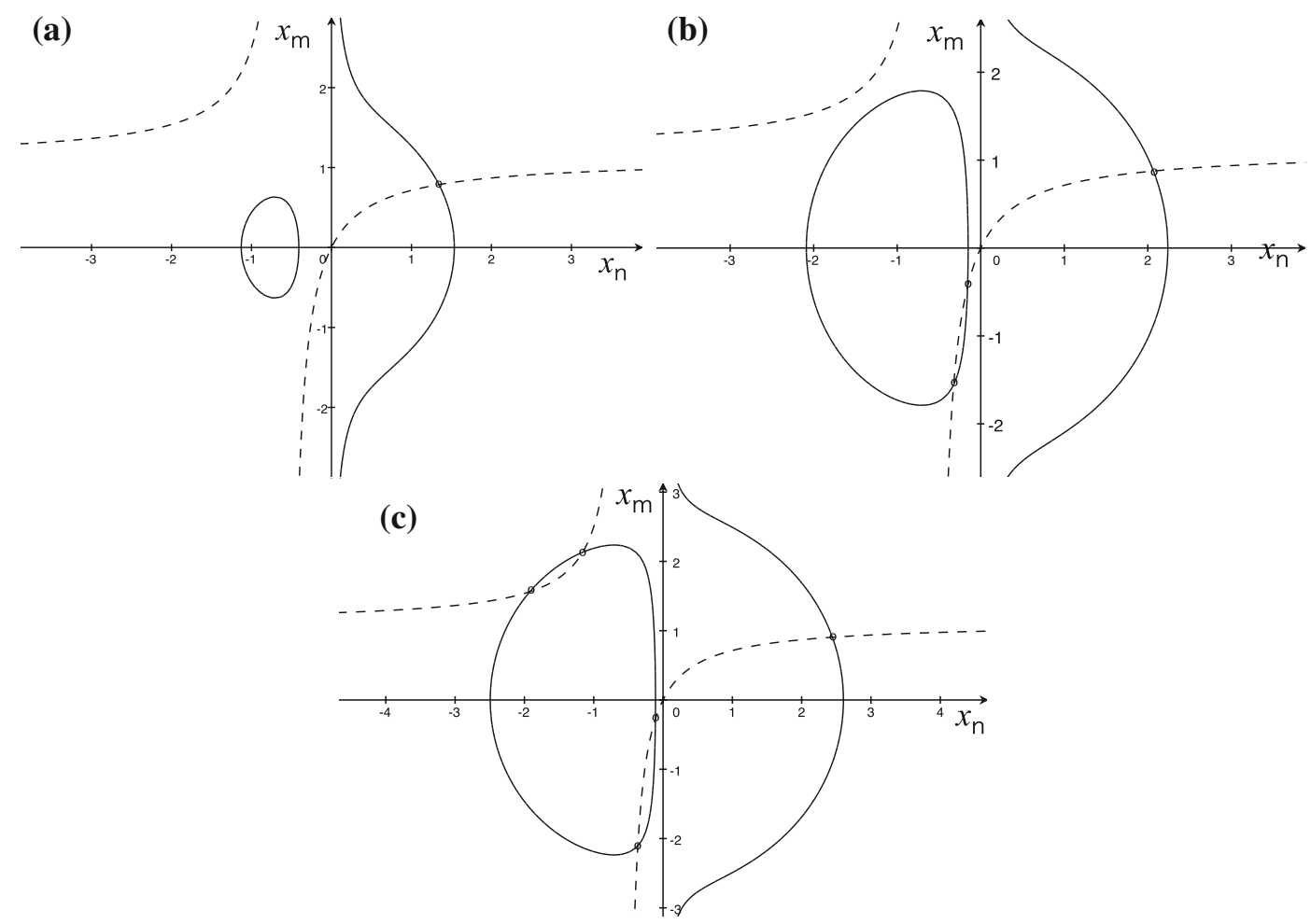

Fig. 5 Solutions $\left(x_{n}, x_{m}\right)$ to system (30) are represented as intersections (circles) of a cubical curve (solid) and a hyperbola (dashed). Parameters are such that $\beta_{n}^{0}=-1 / 2, \beta_{m}^{0}=-2.3$ and $\Phi_{m}=2 \Phi_{n}=-\sqrt{2}$. a $\beta=-2.4, \mathbf{b} \beta=-5.2$ and $\mathbf{c} \beta=-7$
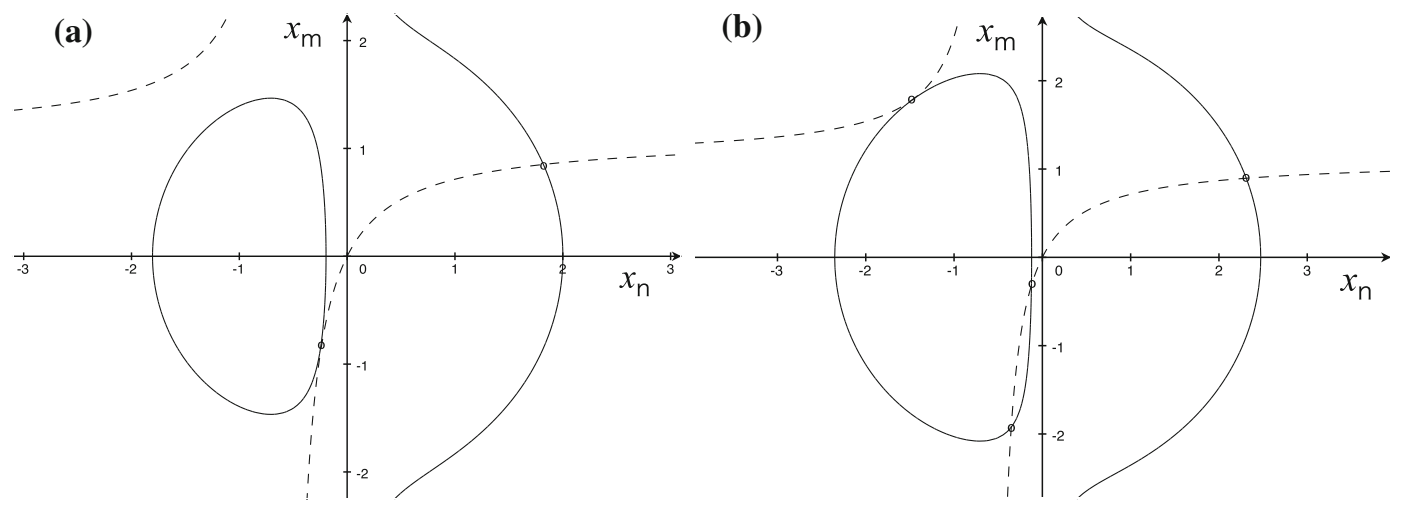

Fig. 6 Geometrical characterization of critical values for $\beta$ when $\beta_{n}^{0}=-1 / 2, \beta_{m}^{0}=-2.3$ and $\Phi_{m}=2 \Phi_{n}=-\sqrt{2}$. a $\beta=\tilde{\beta}_{n m} \approx-3.65$ and $\mathbf{b} \beta=\hat{\beta}_{n m} \approx-5.83$ 
Fig. 7 Bifurcations of bimodal solutions in the non homogeneous case.

Parameters are chosen as in Figs. 5 and 6 . The pitchfork curve corresponding to $\phi_{n}=0$ is in gray

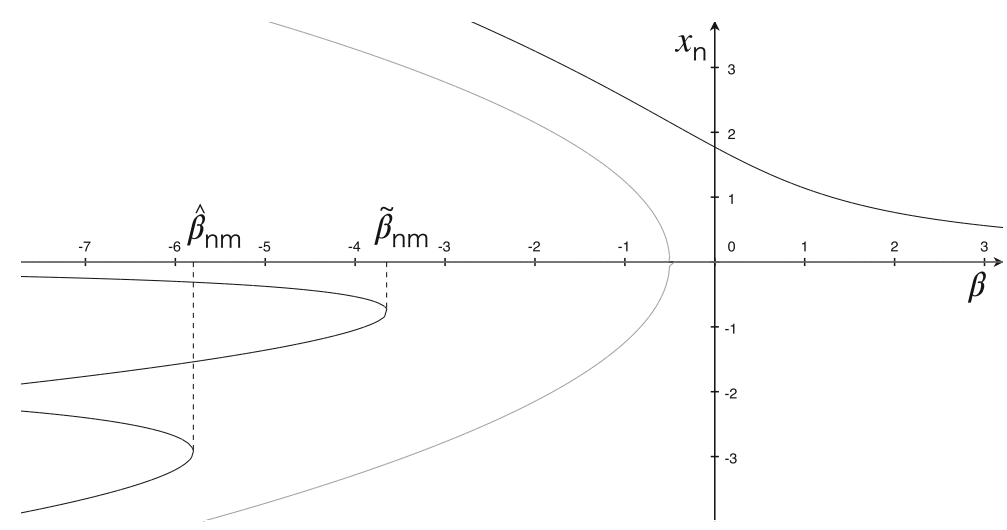

by virtue of (17) and the change of variables (27) we obtain

$$
\left\{\begin{array} { c } 
{ x _ { n } ^ { 3 } + x _ { n } x _ { m } ^ { 2 } + x _ { n } x _ { s } ^ { 2 } + ( \beta - \beta _ { n } ^ { 0 } ) x _ { n } = \Phi _ { n } , } \\
{ x _ { m } ^ { 3 } + x _ { m } x _ { n } ^ { 2 } + x _ { m } x _ { s } ^ { 2 } + ( \beta - \beta _ { m } ^ { 0 } ) x _ { m } = \Phi _ { m } , } \\
{ x _ { s } ^ { 3 } + x _ { s } x _ { n } ^ { 2 } + x _ { s } x _ { m } ^ { 2 } + ( \beta - \beta _ { s } ^ { 0 } ) x _ { s } = \Phi _ { s } , }
\end{array} \quad \left\{\begin{array}{c}
y_{n}=\frac{k x_{n}}{\left(\eta \lambda_{n}+k\right)}, \\
y_{m}=\frac{k x_{m}}{\left(\eta \lambda_{m}+k\right)}, \\
y_{s}=\frac{k x_{s}}{\left(\eta \lambda_{s}+k\right)} .
\end{array}\right.\right.
$$

Since we are looking for nontrivial amplitudes $\alpha_{n}, \alpha_{m}, \alpha_{s}$, system (32) 1 may be rewritten as follows

$$
\left\{\begin{array}{l}
x_{n}^{3}+x_{n} x_{m}^{2}+x_{n} x_{s}^{2}+\left(\beta-\beta_{n}^{0}\right) x_{n}=\Phi_{n}, \\
\frac{\Phi_{m}}{x_{m}}-\frac{\Phi_{n}}{x_{n}}=\beta_{n}^{0}-\beta_{m}^{0}, \\
\frac{\Phi_{s}}{x_{s}}-\frac{\Phi_{n}}{x_{n}}=\beta_{n}^{0}-\beta_{s}^{0} .
\end{array}\right.
$$

In the special case when $(\kappa, \omega) \in \mathcal{R}_{n s}$ we have $\beta_{n}^{0}=$ $\beta_{s}^{0}$ and $\Phi_{n} x_{s}=\Phi_{s} x_{n}$. When $\phi_{n}=\phi_{s}=0$ we recover (31) and then we may state the same conclusions as at the end of the previous subsection. Otherwise, either $\phi_{n}$ or $\phi_{s}$ does not vanish. Assuming for instance $\phi_{n} \neq 0$, then $x_{s}=\Phi_{s} x_{n} / \Phi_{n}$ and system (33) reduces to

$$
\left\{\begin{array}{l}
\left(1+\Phi_{s}^{2} / \Phi_{n}^{2}\right) x_{n}^{3}+x_{n} x_{m}^{2}+\left(\beta-\beta_{n}^{0}\right) x_{n}=\Phi_{n}, \\
\frac{\Phi_{m}}{x_{m}}-\frac{\Phi_{n}}{x_{n}}=\beta_{n}^{0}-\beta_{m}^{0} .
\end{array}\right.
$$

This system looks like (30) and then it may be scrutinized in the same way. Analogous results hold when $(\kappa, \omega) \in \mathcal{R}_{m s}$ and $(\kappa, \omega) \in \mathcal{R}_{n m}$.
In the general case, when $\beta_{n}^{0}, \beta_{m}^{0}, \beta_{s}^{0}$ are all distinct, system (33) leads to a polynomial equation of degree seven in one of the three amplitudes. Depending on the choice of structural parameters and transversal load amplitudes, it admits at most seven distinct triples $\left(x_{n}, x_{m}, x_{s}\right)$ of real solutions. In particular, when $\phi_{n}=$ 0 then

$$
\left\{\begin{array}{l}
{\left[x_{n}^{2}+\beta-\beta_{n}^{0}+\frac{\Phi_{m}^{2}}{\left(\beta_{n}^{0}-\beta_{m}^{0}\right)^{2}}+\frac{\Phi_{s}^{2}}{\left(\beta_{n}^{0}-\beta_{s}^{0}\right)^{2}}\right] x_{n}=0,} \\
x_{m}=\frac{\Phi_{m}}{\beta_{n}^{0}-\beta_{m}^{0}}, \\
x_{s}=\frac{\Phi_{s}}{\beta_{n}^{0}-\beta_{s}^{0}},
\end{array}\right.
$$

so that $x_{m}$ and $x_{s}$ are independent of $\beta$ whereas $x_{n}$ undergoes a pitchfork bifurcation at

$$
\bar{\beta}_{n m}=\beta_{n}^{0}-\frac{\Phi_{m}^{2}}{\left(\beta_{n}^{0}-\beta_{m}^{0}\right)^{2}}-\frac{\Phi_{s}^{2}}{\left(\beta_{n}^{0}-\beta_{s}^{0}\right)^{2}} .
$$

The same occurs when $\phi_{m}=0$ or $\phi_{s}=0$, to within some index exchange.

\section{Discussion}

Depending on the axial-load parameter $\beta$, simple (unimodal) free equilibria exhibit a sequence of pitchfork bifurcations at $\beta_{n}^{0}$, where $n \in \mathbb{N}$ denotes the eigenfunction (mode) which is involved. From (17), these bifurcation values depend on $(\delta, \eta, k)$, the stiffness of the three structural elements forming the system (beam, cable, suspenders). A special choice of 
these parameters lead to the overlapping of different bifurcation values. For instance, the case when $\beta_{n}^{0}=$ $\beta_{m}^{0}$ is referred to as $n: m$ resonance.

Unlike cable-stayed beam systems, where one parameter (the stiffness ratio) is involved in resonances, here the cable-suspended beam we scrutinized involves two distinct parameters, $\omega$ and $\kappa$, which are related to $(\delta, \eta, k)$ [see Eq. (18)]. As a consequence, our discussion about the resonance depends both on $\omega$ and $\kappa$, so that it may be referred to as biparametric resonance.

According to Remark 2, bimodal free equilibria are merely resonant solutions, and they occur only if the structural parametric pair $(\omega, \kappa)$ belongs to $\mathcal{R}$. Moreover, by virtue of (22) and $\lambda_{m} / \kappa>0$, we have

$0<\lambda_{n}<\omega-\kappa$.

For a given pair $(\omega, \kappa)$ such that $\omega>\kappa$, this inequality means that $n: m$ resonances $(n<m)$ may occur only if the $n$-th eigenvalue does not exceed the difference $\omega-\kappa$.

It is apparent from Fig. 2 that no resonant mode occurs when either $\omega \leq \kappa$ (namely, $\eta^{2} \leq k \delta$ ) or $\omega=\eta / \delta<\omega_{12}=\left(\sqrt{\lambda_{1}}+\sqrt{\lambda_{2}}\right)^{2}$. Then, for a given set of structural parameters $\delta, \eta, k$, we have to discuss these inequalities in light of the modeling analysis devised in Sect. 2.1. By mimicking structural parameters and sizes of a single span suspension bridge, it is reasonable to assume

$$
\begin{aligned}
& E \approx E_{c} \approx E_{s}, \quad \frac{\gamma}{\ell} \approx \frac{h}{\ell}, \quad \frac{d}{\ell} \approx \sqrt{\frac{h}{\ell}} \\
& \frac{\left|\Omega_{c}\right|}{|\Omega|} \approx \frac{h}{\ell}, \quad \frac{\left|\Omega_{s}\right|}{|\Omega|} \approx \frac{h^{2}}{\ell^{2}} .
\end{aligned}
$$

Due to the modeling assumptions, we may introduce a reference smallness parameter $\epsilon=h / \ell \ll 1$ (for instance, $\epsilon \approx 10^{-2}$ ). Then, we obtain the following size estimates

$$
\begin{aligned}
& \frac{d}{\ell} \approx \sqrt{\epsilon}, \quad \delta \approx \epsilon^{2}, \quad \eta \approx \frac{1}{\epsilon}, \quad k \approx \frac{1}{\sqrt{\epsilon}}, \\
& \beta \approx \epsilon .
\end{aligned}
$$

Accordingly, we have $\kappa \approx \sqrt{\epsilon}$ and $\omega \approx \epsilon^{-3}$, so that $\omega \gg \kappa, \quad \omega \gg \omega_{12}$.
In the simple case $\lambda_{n}=n^{2} \pi^{2}$, then $\omega_{12}=9 \pi^{2} \approx 10^{2}$. In addition, it is worth noting that $1: 4$ and $2: 3$ resonances arise at the same critical value for $\omega$, namely $\omega_{14}=\omega_{23}=25 \pi^{2}$, but at different values of $\kappa$. This behavior is common to all $n_{1}: n_{2}$ resonances such that $n_{1}+n_{2}=n$ for any fixed $n \in \mathbb{N}, n \geq 5$.

When a distributed (stationary) transversal load is applied, the stationary problem is more complex. For unimodal solutions we observe, as expected under perturbation, the breakdown of the pitchfork bifurcations obtained in free equilibria (see Fig. 3). The corresponding bifurcation value, $\beta_{n}^{\phi}$, is smaller than $\beta_{n}^{0}$ and depends on the transversal load component $\phi_{n}$. Using geometrical arguments, we study the behavior of bimodal steady solutions. When structural parameters are such that $(\omega, \kappa) \in \mathcal{R}$, we argue that each component of the bimodal solution undergoes a perturbed pitchfork bifurcation which is very close to that of unimodal solutions, but occurs at $\tilde{\beta}_{n m}<\min \left\{\beta_{n}^{\phi}, \beta_{m}^{\phi}\right\}$. On the other hand, if $(\omega, \kappa) \notin \mathcal{R}$ each component of the bimodal solution exhibit a more complex pitchfork bifurcation where two different bifurcation values, $\hat{\beta}_{n m}$ and $\tilde{\beta}_{n m}$, are involved (see Fig. 7).

\section{Conclusions}

In this paper the equilibria of an elastically-coupled cable-suspended beam system, as simplified suspension bridge model, is investigated. The beam is assumed to be extensible and subject to a compressive axial load. Indeed, when the road-bed has sectional dimensions which are negligible in comparison with its length (span), then it can be simply modeled as a vibrating one-dimensional beam and this entails that the torsional motion can be ignored. In addition, by neglecting the influence of the towers and side parts of the bridge, the beam may be assumed to have simply supported ends. No longitudinal tension is imposed on the cable since we model it as an elastic string with fixed ends. Unlike cable-stayed beam systems, the suspended beam acts on the cable just through the suspenders (a distributed system of linear springs), so yielding a transversal distributed load on it.

The global analysis of the longtime dynamics of dissipative systems like elastically-coupled cablesuspended beam models, strongly depends on the 
complexity of the steady-states set. In general, when multiple equilibria occur, dissipativity leads the existence of an absorbing set, a closed set which contains all steady states and absorbs trajectories in a finite time. In most cases, it is possible to prove the existence of a global regular attractor of solutions. These arguments motivate the interest to count and possibly construct steady solutions. In particular, the number of such solutions strongly depends on the values of the control parameter $\beta$, in that it is able to induce buckling.

When no vertical load is applied, necessary and sufficient conditions in order to have nontrivial solutions of the stationary problem are established. Their explicit closed-form expressions are found. The steady states are shown to exhibit at most two nonvanishing Fourier modes and the critical values of the axial-load parameter which produce their pitchfork bifurcation (buckling) are established. Depending on two dimensionless parameters, the complete set of resonant modes is devised. In Fig. 2 a sketch of the biparametric resonant set is depicted. When a distributed transversal load is applied to the beam breakdown of the pitchfork bifurcations under perturbation is observed and both unimodal and bimodal stationary solutions are studied in detail. Finally, the more complex behavior occurring when trimodal solutions are involved is briefly sketched.

\section{Compliance with ethical standards}

Conflict of interest The authors declare that they have no conflict of interest.

\section{References}

1. Abdel-Ghaffar AM, Rubin LI (1983) Non linear free vibrations of suspension bridges: theory. ASCE J Eng Mech 109:313-329

2. Abdel-Ghaffar AM, Rubin LI (1983) Non linear free vibrations of suspension bridges: application. ASCE J Eng Mech 109:330-345

3. Ahmed NU, Harbi H (1998) Mathematical analysis of dynamic models of suspension bridges. SIAM J Appl Math 58:853-874

4. Amer YA, Hegazy UH (2012) Chaotic vibration and resonance phenomena in a parametrically excited string-beam coupled system. Meccanica 47:969-984

5. An Y (2002) Nonlinear perturbations of a coupled system of steady state suspension bridge equations. Nonlinear Anal 51:1285-1292
6. Bochicchio I, Vuk E (2010) Longtime behavior of an extensible elastic beam on a viscoelastic foundation. Math Comp Model. 51:833-846

7. Bochicchio I, Giorgi C, Vuk E (2010) Long-term damped dynamics of the exstensible suspension bridge. Int J Differ Eq 2010:383-420

8. Bochicchio I, Giorgi C, Vuk E (2012) Long-term dynamics of the coupled suspension bridge system. Math Models Methods Appl Sci 22:1250021

9. Bochicchio I, Giorgi C, Vuk E (2013) Asymptotic dynamics of nonlinear coupled suspension bridge equations. J Math Anal Appl 402:319-333

10. Bochicchio I, Giorgi C, Vuk E (2014) Long-term dynamics of a viscoelastic suspension bridge. Meccanica 49:2139-2151

11. Bochicchio I, Giorgi C, Vuk E (2014) On the viscoelastic coupled suspension bridge. Evol Eq Control Theory 3:373-397

12. Bochicchio I, Giorgi C, Vuk E (2015) Well-posedness and longtime behaviour of a coupled nonlinear system modeling a suspension bridge. Meccanica 50:665-673

13. Choi QH, Jung T (1999) A nonlinear suspension bridge equation with nonconstant load. Nonlinear Anal 35:649-668

14. Coti Zelati M, Giorgi C, Pata V (2010) Steady states of the hinged extensible beam with external load. Math Models Methods Appl Sci 20:43-58

15. Drábek P, Holubová G, Matas A, Nečesal P (2003) Nonlinear models of suspension bridges: discussion of the results. Appl Math 48:497-514

16. Dell'Oro F, Giorgi C, Pata V (2015) Asymptotic behavior of coupled linear systems modeling suspension bridges. Z Angew Math Phys 66:1095-1108

17. Gattulli V, Lepidi M (2003) Nonlinear interactions in the planar dynamics of cable-stayed beam. Int J Solids Struct 40:4729-4748

18. Gattulli V, Lepidi M (2007) Localization and veering in cable-stayed bridge dynamics. Comput Struct 85(21-22):1661-1668

19. Gattulli V, Lepidi M, John HG, Taylor AC (2005) One-totwo global interaction in a cable-stayed beam observed through analytical, finite element and experimental models. Int J Nonlinear Mech 40:571-588

20. Gattulli V, Morandini M, Paolone A (2002) A parametric analytical model for non-linear dynamics in cable-stayed beam. Earthq Eng Struct Dyn 31:1281-1300

21. Giorgi C, Naso MG (2011) Modeling and steady states analysis of the extensible thermoelastic beam. Math Comp Model 53:896-908

22. Hale JK (1988) Asymptotic behavior of dissipative systems. American Mathematical Society, Providence

23. Holubová G, Matas A (2003) Initial-boundary problem for the nonlinear string-beam system. Math Anal Appl 288:784-802

24. Lacarbonara W (2013) Nonlinear structural mechanics. Theory, dynamical phenomena and modeling. Springer, New York

25. Lazer AC, McKenna PJ (1990) Large-amplitude periodic oscillations in suspension bridges: some new connections with nonlinear analysis. SIAM Rev. 32:537-578 
26. McKenna PJ (2014) Oscillations in suspension bridges, vertical and torsional. Discrete Contin Dyn Syst Ser S 7:785-791

27. McKenna PJ, Walter W (1987) Nonlinear oscillations in a suspension bridge. Arch Rational Mech Anal 98:167-177

28. Reiss EL, Matkowsky BJ (1971) Nonlinear dynamic buckling of a compressed elastic column. Quart Appl Math 29:245-260
29. Wang ZQ, Sun CS, Zhao YB, Yi ZP (2014) Modeling and nonlinear modal characteristics of the cable-stayed beam. Eur J Mech A-Solid 47:58-69

30. Wei MH, Xiao YQ, Liu HT (2012) Bifurcation and chaos of a cable-beam coupled system under simultaneous internal and external resonances. Nonlinear Dyn 67:1969-1984

31. Woinowsky-Krieger S (1950) The effect of an axial force on the vibration of hinged bars. J Appl Mech 17:35-36 